\title{
Tunable Fermi acceleration in the driven elliptical billiard
}

\author{
F. Lenz, ${ }^{1, *}$ F.K. Diakonos, ${ }^{2}$ and P. Schmelcher ${ }^{1,3}$ \\ ${ }^{1}$ Physikalisches Institut, Universität Heidelberg, \\ Philosophenweg 12, 69120 Heidelberg, Germany \\ ${ }^{2}$ Department of Physics, University of Athens, GR-15771 Athens, Greece \\ ${ }^{3}$ Theoretische Chemie, Physikalisch-Chemisches Institut, \\ Universität Heidelberg, Im Neuenheimer Feld 229, 69120 Heidelberg, Germany
}

(Dated: October 26, 2018)

\begin{abstract}
We explore the dynamical evolution of an ensemble of non-interacting particles propagating freely in an elliptical billiard with harmonically driven boundaries. The existence of Fermi acceleration is shown thereby refuting the established assumption that smoothly driven billiards whose static counterparts are integrable do not exhibit acceleration dynamics. The underlying mechanism based on intermittent phases of laminar and stochastic behavior of the strongly correlated angular momentum and velocity motion is identified and studied with varying parameters. The diffusion process in velocity space is shown to be anomalous and we find that the corresponding characteristic exponent depends monotonically on the breathing amplitude of the billiard boundaries. Thus it is possible to tune the acceleration law in a straightforwardly controllable manner.
\end{abstract}

PACS numbers: 05.45.-a,05.45.Ac,05.45.Pq 
Fermi acceleration was first proposed in 1949 [1] to explain the high energies of cosmic ray particles interacting with a time-dependent magnetic field (for a review see [2]). Nowadays, Fermi acceleration is investigated in a variety of systems belonging to different areas of physics, such as astrophysics [3, 4, 5], plasma physics [6, 7], atom optics [8, 9] and has even been used for the interpretation of experimental results in atomic physics [10]. In the context of dynamical system theory, Fermi acceleration is defined as the unlimited growth of the energy of a particle moving in a time-dependent potential. For the experimental realization of Fermi acceleration in the laboratory, driven, i.e. time-dependent billiards are the most promising devices. A particle propagating in these systems gains or looses energy whenever it hits the billiards' moving boundary. An infinite sequence of such collisional events can in principle lead to Fermi acceleration of the scattered particle. Driven billiards can be realized experimentally in various ways, such as (superconducting) mesoscopic cavities (see ref. [11, 12] and refs. therein) or atom optical setups employing acousto-optical scanners for the (time-dependent) deflection of correspondingly detuned laser beams [13].

The simplest realization of a driven billiard is the well-known Fermi-Ulam model (FUM) [14, 15, 16] which consists of an ensemble of non-interacting freely moving particles in one dimension bouncing between an oscillating and a fixed wall. The FUM and its variants have been the subject of extensive theoretical (see Ref. [15] and references therein) and experimental [17, 18, 19] studies. It has been proven [16] that a necessary condition for the development of Fermi acceleration in the FUM is the non-smooth (non-differentiable) dependence of the velocity of the moving wall on time (this includes especially randomized dynamical systems). An alternative scenario for the occurrence of Fermi acceleration in $1 D$ systems is a bouncer model [20] with an additional nonlinear time-independent term in the potential.

In contrast to the $1 D$ case, driven billiards with a higher dimensional configuration space pose many open questions. The lack of the possibility to visualize phase space in terms of Poincaré surfaces of section complicates the analysis of e.g. $2 D$ time-dependent billiards [21]. Despite this fact, several studies of Fermi acceleration in these systems [22, 23, 24, 25] have been performed. These investigations provide us with the conclusion that a sufficient condition for the occurrence of Fermi acceleration in a $2 D$ smoothly driven billiard is the existence of a chaotic part in the phase space of the corresponding time-independent system, obtained by assuming static boundaries (see the LRA conjecture in ref. [23]). This is 
supported by the absence of Fermi acceleration in the smoothly oscillating circular billiard where the corresponding static system is integrable [26]. On the other hand the static Lorentz gas possesses a predominantly chaotic phase space and its harmonically driven counterpart exhibits Fermi acceleration [24, 27]. Similar results were obtained for the oscillating stadiumlike [28], oval [29] as well as annular billiard [25], each of them possessing chaotic portions with respect to the phase space of the corresponding time-independent system.

To elucidate the above-described problem of the existence of Fermi acceleration for driven billiards whose time-independent counterparts are integrable, we investigate here the elliptical harmonically driven billiard. According to the existing literature the ellipse should not exhibit Fermi acceleration (see e.g. ref. [22]). We show that, opposite to what is expected in the literature, Fermi acceleration occurs for ensembles of particles propagating in a breathing elliptical billiard even for very small oscillation amplitudes. The driving causes a layer of unstable motion around the separatrix of the elliptical billiard leading to large fluctuations of the velocities of the particles as they cross it. These fluctuations, which increase with time, lead to an anomalous diffusion in velocity space and an unlimited growth of the mean kinetic energy of the multiple scattered particles. The anomalous behavior of the velocity diffusion process is due to the intermittent character of the dynamics attributed to the instability of the fixed points in the regime of librators. We determine the dependence of the exponent of the acceleration law on the amplitude of the oscillation of the boundary leading to the conclusion that the smoothly driven elliptical billiard represents a tunable source of Fermi acceleration.

Let us specify our setup (for a more detailed description see ref. [31]). The static ellipse is integrable: besides the energy $E$ the product of the angular momenta $F$ about the two foci of the ellipse [30] is conserved.

$$
F(\varphi, p)=\frac{p^{2}\left(1+\left(1-\varepsilon^{2}\right) \cot ^{2} \varphi\right)-\varepsilon^{2}}{1+\left(1-\varepsilon^{2}\right) \cot ^{2} \varphi-\varepsilon^{2}},
$$

where $\epsilon$ is the numerical eccentricity. The variables $p=\cos \alpha$ and $\varphi$ are defined at the elliptical boundary of the billiard: $\alpha$ is the angle between the tangent and the trajectory of the colliding particle while $\varphi$ is the azimuthal angle, see eq. (21) and Fig. 1. The phase space is globally divided by the separatrix $(F=0)$, associated with two hyperbolic fixed points, into rotators $(F>0)$ and librators $F<0$. Two elliptic fixed points are located at the 
global minimum $F_{\min }$ of $F$. The driving law of the elliptic boundary is:

$$
\left(\begin{array}{l}
x(t) \\
y(t)
\end{array}\right)=\left(\begin{array}{c}
\left(A_{0}+C \sin (\omega t)\right) \cos \varphi \\
\left(B_{0}+C \sin (\omega t)\right) \sin \varphi
\end{array}\right)
$$

where $t$ is time, $(x(t), y(t))$ is a point on the boundary, $\varphi$ is a $2 \pi$-periodic parameter, $C>0$ is the driving amplitude, $\omega$ is the frequency of oscillation and $A_{0}, B_{0}$ are the equilibrium values of the long and the short half-diameter, respectively. The phase of oscillation at $t=0$ is set to zero in the following. In order to reduce the number of parameters to be varied in our investigations, we fix $\omega=1, A_{0}=2$ and $B_{0}=1$ (arbitrary units). The scattering dynamics in the billiard is described by an implicit $4 D$ map specifying the sequence of the collisional events on the boundary of the ellipse. A convenient choice of the variables of this map is $\left(\varphi_{n}, p_{n},\left|\vec{v}_{n}\right|, \xi_{n}\right)$, where $\left|\vec{v}_{n}\right|$ is the magnitude of particles' velocity after the collision and $\xi_{n}$ is the phase of the boundary oscillation at the $n$-th collision. The implicit equations defining this map are:

$$
\begin{gathered}
\left(\frac{v_{n}^{x}\left(t_{n+1}-t_{n}\right)+x_{n}}{A_{0}+C \sin \left(\omega t_{n+1}\right)}\right)^{2}+\left(\frac{v_{n}^{y}\left(t_{n+1}-t_{n}\right)+y_{n}}{B_{0}+C \sin \left(\omega t_{n+1}\right)}\right)^{2}-1=0 \\
\boldsymbol{x}_{n+1}=\boldsymbol{x}_{n}+\boldsymbol{v}_{n}\left(t_{n+1}-t_{n}\right) \\
\boldsymbol{v}_{n+1}=\boldsymbol{v}_{n}-2\left[\hat{\boldsymbol{n}}_{n+1} \cdot\left(\boldsymbol{v}_{n}-\boldsymbol{u}_{n+1}\right)\right] \cdot \hat{\boldsymbol{n}}_{n+1}
\end{gathered}
$$

where the smallest $t_{n+1}>t_{n}$ that solves (3a) has to be taken and $\xi_{n+1}=t_{n+1} \bmod 2 \pi$. $\boldsymbol{x}_{n}=\left(x_{n}, y_{n}\right)$ is the $n$th collision point. $\varphi_{n+1}$ can be obtained by inverting (2). In eq. (3c), $\boldsymbol{u}_{n+1}$ is the boundary velocity and $\hat{\boldsymbol{n}}_{n+1}$ the normal vector of the collisional event occurring at time $t_{n+1}$ and position $\boldsymbol{x}_{n+1}$. When iterating the mapping (3) numerically, solving (3a) requires the major computational effort in terms of CPU-time, even when applying indispensable advanced bracketing techniques. When the driving is applied, both $E$ and $F$ are no longer conserved quantities. However, they are still very valuable for the description of the dynamics in the presence of the driving (2), since at every time instant the elliptical form of the boundary is preserved.

Before discussing the ensemble averaged properties of the time-dependent system, it is illuminating to analyze a typical trajectory being initially on a librator orbit $(C=0.2)$. Fig. 2 shows the evolution of $F$ (upper curve, light gray) and $|\vec{v}|$ (lower curve, light gray) respectively as a function of the number of collisions $n$. According to Fig. 2 a,$F(n)$ alternates between periods of regular (laminar phases) oscillations (intervals $\left[0,2.8 \cdot 10^{5}\right]$ and $[1.4$. 


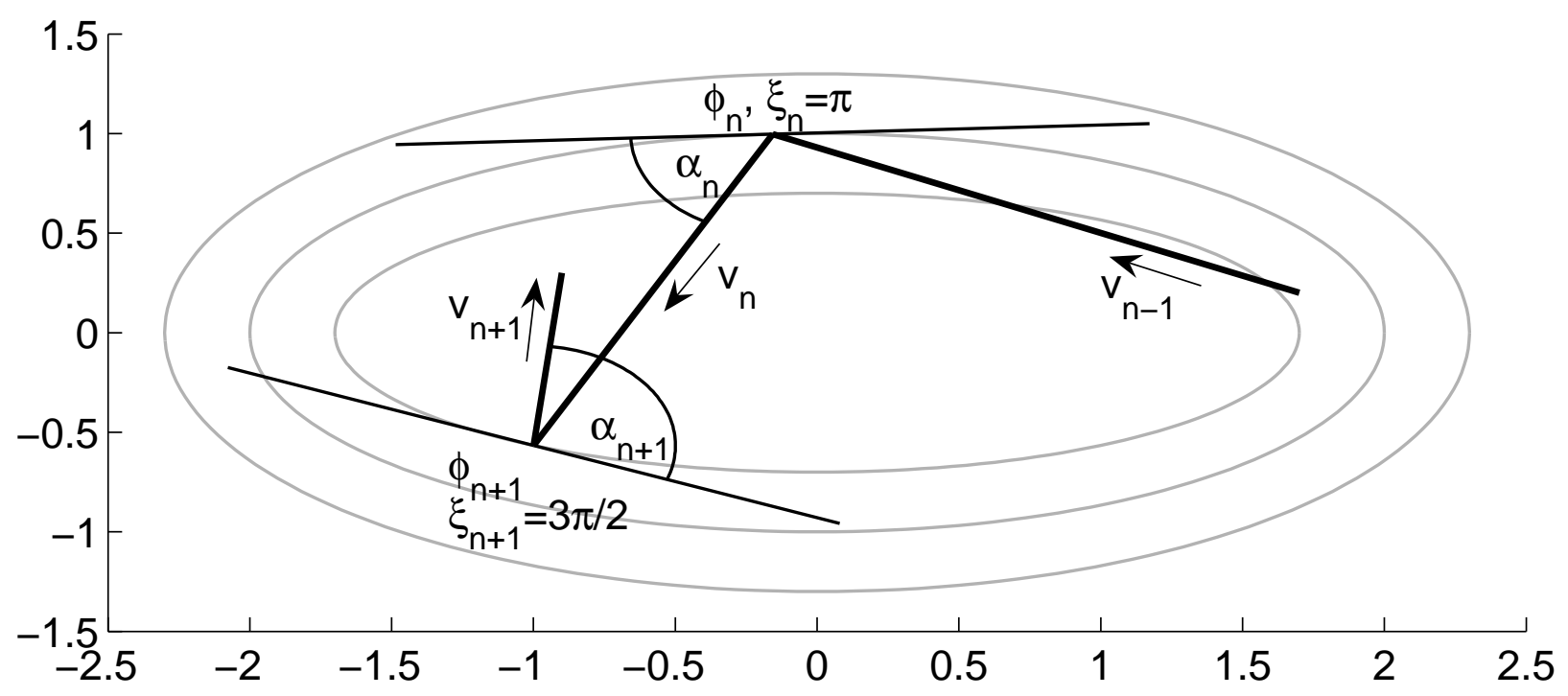

FIG. 1: Schematic picture of the setup of the elliptical billiard.

$\left.10^{6}, 1.75 \cdot 10^{6}\right]$ ) and periods of irregular fluctuations (turbulent phases). During these laminar phases, $F(n)$ never crosses the $F=0$ line, whereas during the turbulent phases $F(n)$ remains essentially within the zone $\left[F_{\min }, F_{\max }\right]=[-1,0.4]$, repeatedly crossing the $F=0$ line associated with the separatrix (the separatrix is defined by $F=0$, independent of $t$ ). From Fig. 2b and 2re we see that this structure of laminar and turbulent phases exists on different scales of $n$. Whenever $F(n)$ is in a laminar phase, $|\vec{v}|$ oscillates around a fixed central value, whereas during the turbulent $F(n)$ periods, $|\vec{v}|(n)$ starts to develop intervals with strong, irregular fluctuations leading to a sudden increase or decrease of its central value. In fact, the increasing parts of the trajectory $|\vec{v}|(n)$ prevail such that a net increase of the velocity for longer times can be observed.

The above-observed behavior indicates two important dynamical properties of the driven system: (i) the region around the separatrix is characterized by stochastic dynamics and (ii) the trajectories $F(n)$ and $|\vec{v}|(n)$ are strongly correlated (see Fig. 2). The enhanced stochasticity of the dynamics around $F \approx 0$ is also verified by the appearance of the corresponding power-spectrum $S(k)=\frac{2 \pi}{N}\left|\sum_{n=1}^{N} F(n) e^{\frac{-2 \pi i(k-1)(n-1)}{N}}\right|$ presented in Fig. 3. Clearly, the continuous part of the spectrum is dominating in this case (Fig. $3 \mathrm{~b}$ ). On the contrary, in regions of large negative $F$ values the power spectrum, shown in Fig. 3 a, is dominated by isolated peaks while the continuous background is much less pronounced. Furthermore, the correlated behavior of $|\vec{v}|$ and $F$ suggests that an acceleration mechanism may be as- 
sociated with the crossing of the $F=0$ line. To elucidate this, it is necessary to integrate

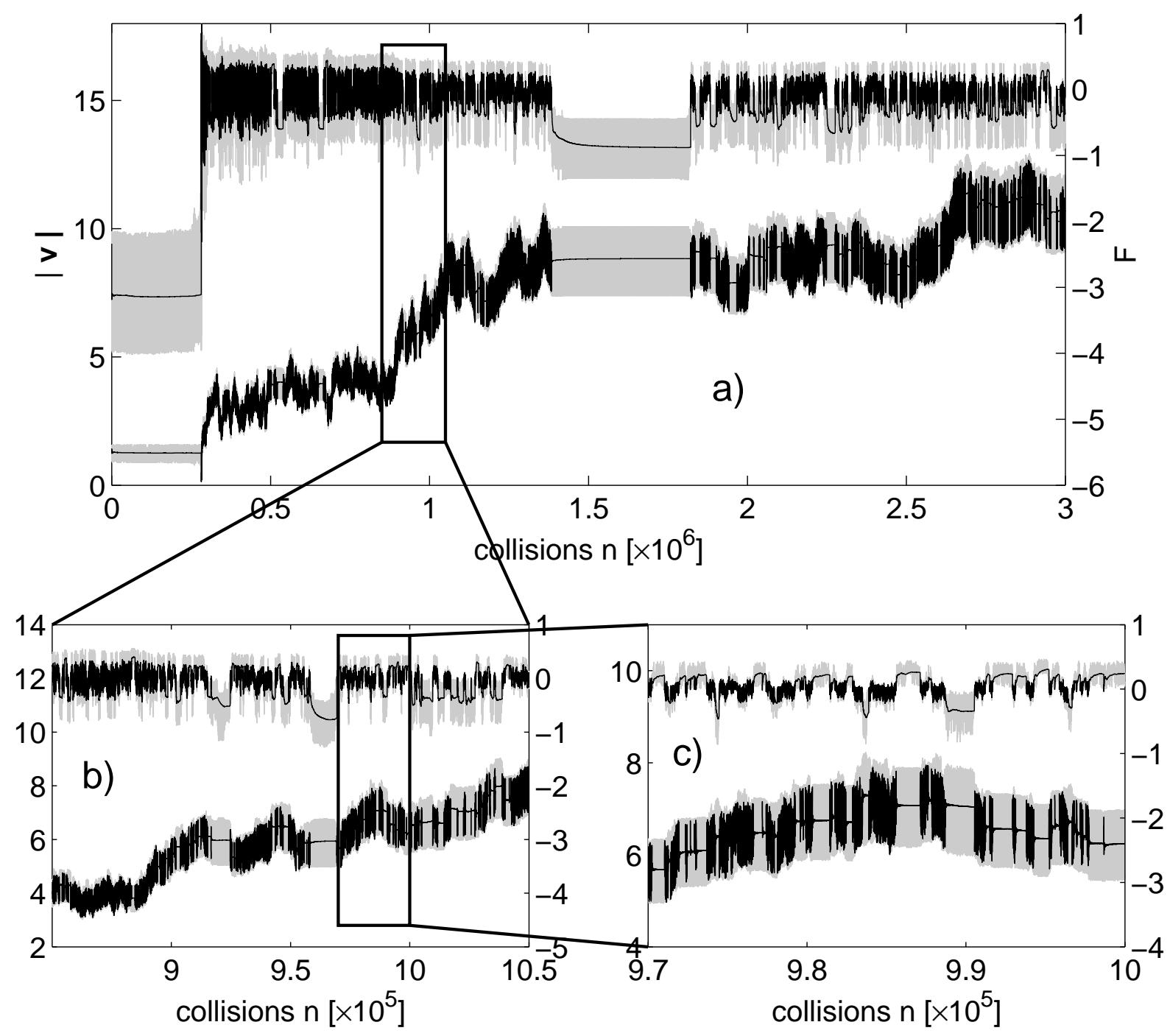

FIG. 2: The functions $F(n)$ (upper curve, light gray) and $|\vec{v}|(n)$ (lower curve, light gray) for a single trajectory for $C=0.2$ in arbitrary units. The solid black lines indicate the cumulative mean in each case. The two successive magnifications demonstrate that the corresponding fluctuations occur on many scales.

out regular as well as stochastic fluctuations of the laminar phase occurring on small time scales. For such an averaging procedure to be successful one has to detect first efficiently the intervals of laminar evolution. A rough estimation of these intervals is obtained by analyzing the timeseries $F(n)$. As 'working intervals' (to calculate the cumulative mean) resembling the laminar phases, we identify the dynamics between two successive zero crossings of $F$. 
Then, in each such interval $j$, we calculate the cumulative mean $\bar{F}(n)_{j}=\frac{1}{n} \sum_{i=1}^{n} F_{i}^{(j)}$ and $|\overline{\vec{v}}|(n)_{j}=\frac{1}{n} \sum_{i=1}^{n}\left|\vec{v}_{i}^{(j)}\right|$ as a function of $n$. Here $n$ takes values $n=1, . ., L_{j}$ with $L_{j}$ being the number of collisions between the $j$-1-th and the $j$-th zero crossing of $F$. The results for $\bar{F}_{j}$ and $|\overline{\vec{v}}|_{j}$ of the above-considered trajectory are shown as solid black lines in Fig. 2 , The similarity of the dynamics of the cumulative mean of $F$ with a typical trajectory showing intermittency [32] is remarkable. Additionally, the one-to-one correspondence between periods of stochastic behavior (bursts) of $\bar{F}_{j}$ and periods of fluctuations of $|\overline{\vec{v}}|_{j}$ is obvious. The intermittent character of the $F$-dynamics goes along with a specific distribution of the laminar lengths $L_{j}$, i.e. the intervals between successive zeros of $F$, which according to the theory of intermittent maps [33] should obey a power-law. Indeed, we find asymptotically (for large values of $L_{j}$ ) a power-law behavior of the distribution with a characteristic exponent of $\eta=-2.00 \pm 0.05$.
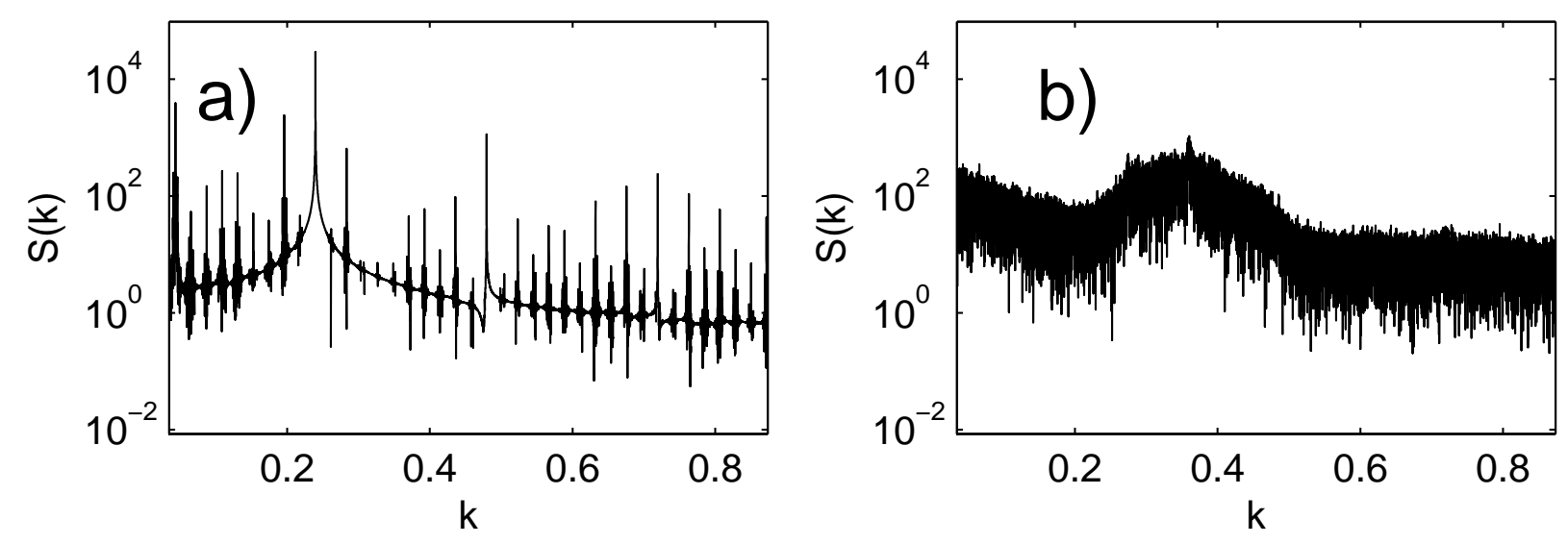

FIG. 3: Power spectrum $S(k)$ of a typical laminar (a) and turbulent phase (b) in arbitrary units.

Let us now investigate whether Fermi acceleration occurs in the driven ellipse by examining $\langle|\vec{v}|\rangle(n)$, where $\langle$.$\rangle denotes ensemble averaging. In Fig. 4$ we show the results of our simulations obtained using 1000 trajectories with initial values of $\alpha$ and $\varphi$ uniformly distributed in $[0, \pi]$ and $[0,2 \pi]$, respectively, for different driving amplitudes $C$ (see also Table I). Each trajectory is propagated $10^{7}$ collisions and possesses the initial velocity $\left|\vec{v}_{0}\right|=1$. Obviously, we encounter Fermi acceleration for all ensembles, i.e. driving amplitudes considered here. After an initial transient behavior of typically $O\left(10^{4}\right)$ collisions characterized by relatively small variations of the mean velocity, the function $\langle|\vec{v}|\rangle(n)$ becomes an increasing power-law $\langle|\vec{v}|\rangle(n) \sim n^{\beta(C)}(\beta(C)>0)$. The associated exponent $\beta$, as displayed in Table 


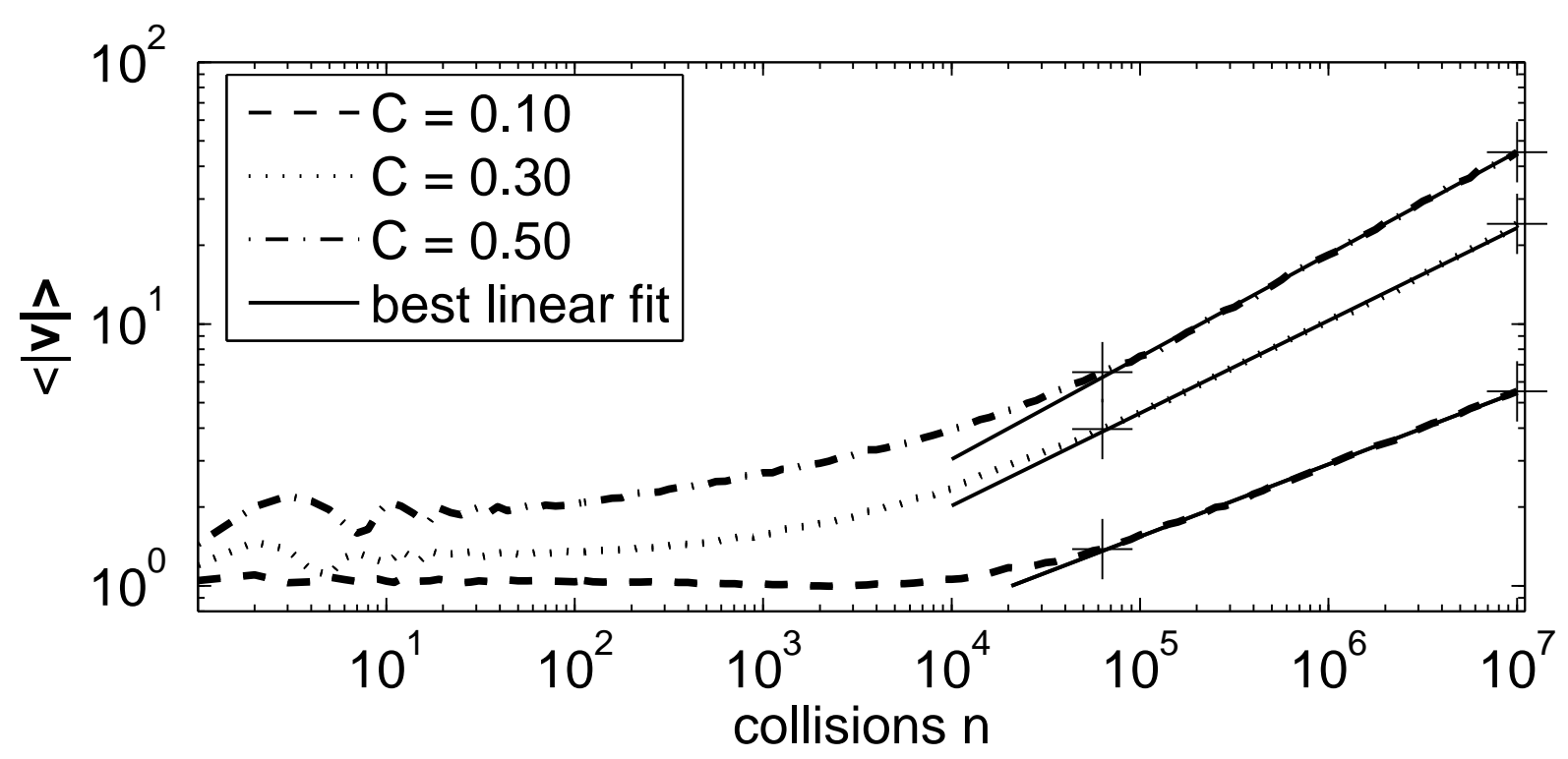

FIG. 4: The function $\langle|\vec{v}|\rangle(n)$ (arbitrary units) for an ensemble of $10^{3}$ trajectories for different values of the driving amplitude $C$, together with the best linear fit (between the two crosses).

TABLE I: Exponent $\beta(C)$ for $\langle|\vec{v}|\rangle(n) \sim n^{\beta(C)}$ for different values of the amplitude $C$.

\begin{tabular}{llllllll}
\hline \hline$C$ & 0.05 & 0.10 & 0.20 & 0.30 & 0.40 & 0.50 & 0.60 \\
$\beta(C)$ & 0.17 & 0.28 & 0.34 & 0.36 & 0.39 & 0.40 & 0.41 \\
\hline \hline
\end{tabular}

I, depends monotonically on $C$. The diffusion in velocity space is anomalous in agreement with the fact that the dynamics in $F$-space is intermittent. This statement holds, of course, not only for $\langle|\vec{v}|\rangle(n)$ but also for the dependence on the real time $\langle|\vec{v}|\rangle(t)$. The above analysis allows us to identify the basic ingredients of the mechanism responsible for Fermi acceleration in the driven elliptical billiard. For $C=0$ the voluminous librator region in phase space contains a continuous set of invariant and impenetrable manifolds. In the presence of the driving $(C \neq 0)$ it becomes penetrable and individual trajectories starting in the librator part of phase space are 'pushed' towards the separatrix (ejection phase). Due to the driving the neighborhood of the separatrix is replaced by a chaotic layer whose properties depend on the amplitude $C$ of the boundary oscillation. While the dynamics is laminar in the ejection phase, the motion in the chaotic layer is to a large extent stochastic, introducing large fluctuations of $F$ and consequently of the velocity which in turn leads to Fermi acceleration.

In conclusion we have investigated the development of Fermi acceleration (FA) in the 
harmonically driven elliptical billiard. Opposite to the expectations according to the stateof-the-art of the field, we discover FA of the form $\langle|\vec{v}|\rangle(n) \sim n^{\beta}$ with the exponent $\beta$ being tunable via the amplitude of the oscillation of the elliptic boundary. We remark that no FA has been observed in Ref. [22] in the driven ellipse. However, Ref. [22] employs a slightly different driving mode. Additionally, the transient in which there is no FA is rather long and could be easily misinterpreted as the absence of FA. Here we demonstrate for the first time that FA is observed in a higher dimensional driven system for which the corresponding static counterpart is integrable. To our knowledge, the considered system is also the first billiard system showing an acceleration law that is tunable in a straightforward and controllable manner despite the complex structure of the underlying higher dimensional phase space. We show that the observed tunable anomalous diffusion in velocity space is associated with an intermittent dynamics of the second integral $(F)$ of the static integrable system, all by all triggered by the driving.

This work was supported by the German Research Foundation (DFG) under the contract Schm885/13-1 and within the framework of the Excellence Initiative through the Heidelberg Graduate School of Fundamental Physics (GSC 129/1). F.L. acknowledges support from the Landesgraduiertenförderung Baden-Württemberg. F.K.D. likes to thank the DAAD for financial support.

* lenz@physi.uni-heidelberg.de

[1] E. Fermi, Phys. Rev. 75, 1169 (1949).

[2] R. Blandford and D. Eichler, Phys. Rep. 154 (1987).

[3] A. Veltri and V. Carbone, Phys. Rev. Lett. 92143901 (2004).

[4] K. Kobayakawa, Y.S. Honda and T. Samura, Phys. Rev. D 66083004 (2002).

[5] M. A. Malkov, Phys. Rev. E 58, 4911 (1998).

[6] G. Michalek, M. Ostrowski and R. Schlickeiser, Sol. Phys. 184, 339 (1999).

[7] A. V. Milovanov and L. M. Zelenyi, Phys. Rev. E 64, 052101 (2001).

[8] F. Saif, I. Bialynicki-Birula, M. Fortunato, W. P. Schleich, Phys. Rev. A 58, 4779 (1998).

[9] A. Steane, P. Szriftgiser, P. Desbiolles, J.Dalibard, Phys. Rev. Lett. 74, 4972 (1995).

[10] G. Lanzano et al, Phys. Rev. Lett. 83, 4518 (1999). 
[11] H.-J. Stöckmann, Quantum Chaos: An Introduction, Cambridge University Press, 1999.

[12] C. Dembowski, H.-D Gräf, A. Heine, T. Hesse, H. Rehfeld, A. Richter, Phys. Rev. Lett. 86, 3284 (2001).

[13] V. Milner, J. L. Hanssen, W. C. Campbell, and M. G. Raizen, Phys. Rev. Lett. 86, 1514 (2001); N. Friedman, A. Kaplan, D. Carasso, and N. Davidson, Phys. Rev. Lett. 86, 1518 (2001).

[14] S. Ulam, in Proceedings of the Fourth Berkley Symposium on Mathematics, Statistics, and Probability, California U.P., Berkeley, 1961, Vol. 3, p. 315.

[15] M. A. Lieberman and A. J. Lichtenberg, Phys. Rev. A 5, 1852 (1972).

[16] A. J. Lichtenberg, M. A. Lieberman, Regular and Chaotic Dynamics, Appl. Math. Sci. 38, Springer Verlag, New York, 1992.

[17] Z. J. Kowalik, M. Franaszek and P. Pieranski, Phys. Rev. A 37, 4016 (1988).

[18] S. Warr et al, Physica A 231, 551 (1996).

[19] S. Celaschi and R. L. Zimmerman, Phys. Lett. A 120, 447 (1987).

[20] L. D. Pustilinikov, Theor. Math. Phys. 57, 1035 (1983); Sov. Math. Dokl. 35(1), 88 (1987); Russ. Acad. Sci. Sb. Math. 82(1), 231 (1995).

[21] J. Koiller, R. Markarian, S. O. Kamphorst and S. P. de Carvalho, Nonlinearity 8, 983 (1995).

[22] J. Koiller, R. Markarian, S. O. Kamphorst and S. P. de Carvalho, J. Stat. Phys. 83, 127 (1996).

[23] A. Yu. Loskutov, A. B. Ryabov, and L. G. Akinshin, J. Exp. Theor. Phys. 89, 966 (1999); A. Yu. Loskutov, L. G. Akinshin, A. N. Sobolevskii, Prikladnaya Nelineinaya Dinamika 9, 50 (2001).

[24] A. Yu. Loskutov, A. B. Ryabov, and L. G. Akinshin, J. Phys. A: Math. Gen. 337973 (2000).

[25] R. E. de Carvalho, F. C. Souza, E. D. Leonel, Phys. Rev. E 73, 66229 (2006); J. Phys. A: Math. Gen. 39, 3561 (2006).

[26] S. O. Kamphorst and S. P. de Carvalho, Nonlinearity 12, 1363 (1999).

[27] A. K. Karlis, P. K. Papachristou, F. K. Diakonos, V. Constantoudis and P. Schmelcher, Phys. Rev. Lett. 97, 194102 (2006).

[28] A. Yu. Loskutov and A. B. Ryabov, J. Stat. Phys. 108, 995 (2002).

[29] E. D. Leonel, J. K. L. da Silva and S. O. Kamphorst, preprint 2006.

[30] M. V. Berry, Eur. J. Phys. 2, 91 (1981). 
[31] F. Lenz, F. K. Diakonos and P. Schmelcher, Europhys. Lett. 79, 20002 (2007).

[32] I. Procaccia and H. Schuster, Phys. Rev. A 28, 1210 (1983).

[33] H. G. Schuster, Deterministic Chaos, VCH Verlagsgesellschaft mbH, Weinheim, 2nd edition, 1989. 